Published in final edited form as:

J Am Chem Soc. 2021 February 24; 143(7): 2930-2937. doi:10.1021/jacs.0c13034.

\title{
The Asymmetric Synthesis of Amines via Nickel-Catalyzed Enantioconvergent Substitution Reactions
}

\author{
Ze-Peng Yang ${ }^{\#}$, \\ Division of Chemistry and Chemical Engineering, California Institute of Technology, Pasadena, \\ California 91125, United States \\ Dylan J. Freas ${ }^{\#}$, \\ Division of Chemistry and Chemical Engineering, California Institute of Technology, Pasadena, \\ California 91125, United States \\ Gregory C. Fu \\ Division of Chemistry and Chemical Engineering, California Institute of Technology, Pasadena, \\ California 91125, United States \\ \# These authors contributed equally to this work.
}

\begin{abstract}
Chiral dialkyl carbinamines are important in fields such as organic chemistry, pharmaceutical chemistry, and biochemistry, serving for example as bioactive molecules, chiral ligands, and chiral catalysts. Unfortunately, most catalytic asymmetric methods for synthesizing dialkyl carbinamines do not provide general access to amines wherein the two alkyl groups are of similar size (e.g., $\mathrm{CH}_{2} \mathrm{R}$ versus $\mathrm{CH}_{2} \mathrm{R}^{1}$ ). Herein, we report two mild methods for the catalytic enantioconvergent synthesis of protected dialkyl carbinamines, both of which use a chiral nickel catalyst to couple an alkylzinc reagent (1.1-1.2 equivalents) with a racemic partner, specifically, an a-phthalimido alkyl chloride or an $\mathrm{N}$-hydroxyphthalimide (NHP) ester of a protected a-amino acid. The methods are versatile, providing dialkyl carbinamine derivatives that bear an array of functional groups. For couplings of NHP esters, we further describe a one-pot variant wherein the NHP ester is generated in situ, allowing the generation of enantioenriched protected dialkyl carbinamines in one step from commercially-available amino acid derivatives; we demonstrate the utility of this method by applying it to the efficient catalytic enantioselective synthesis of a range of interesting target molecules.
\end{abstract}

\section{Graphical Abstract}

\author{
Corresponding Author: Gregory C. Fu - gcfu@ caltech.edu. \\ The authors declare no competing financial interest. \\ ASSOCIATED CONTENT \\ Supporting Information \\ The Supporting Information is available free of charge at https://pubs.acs.org/doi/10.1021/jacs.0c13034. \\ Procedures, characterization data, and additional references (PDF) \\ X-ray crystallographic data for $\mathbf{6}(\mathrm{CIF})$ \\ $\mathrm{X}$-ray crystallographic data for $\mathbf{4 2}(\mathrm{CIF})$ \\ Complete contact information is available at: https://pubs.acs.org/10.1021/jacs.0c13034
}


Method 1: Substitution of alkyl chlorides

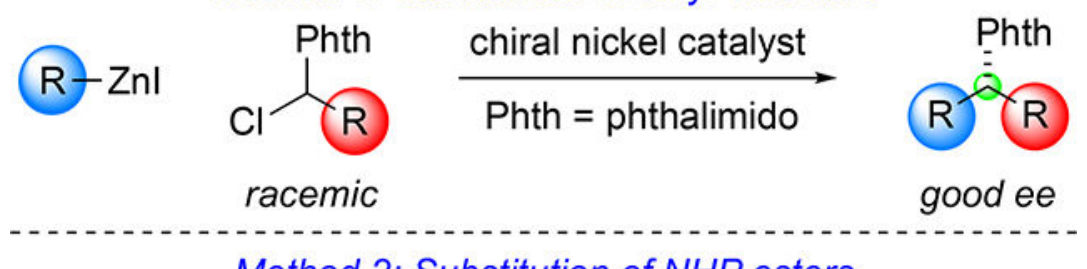

Method 2: Substitution of NHP esters

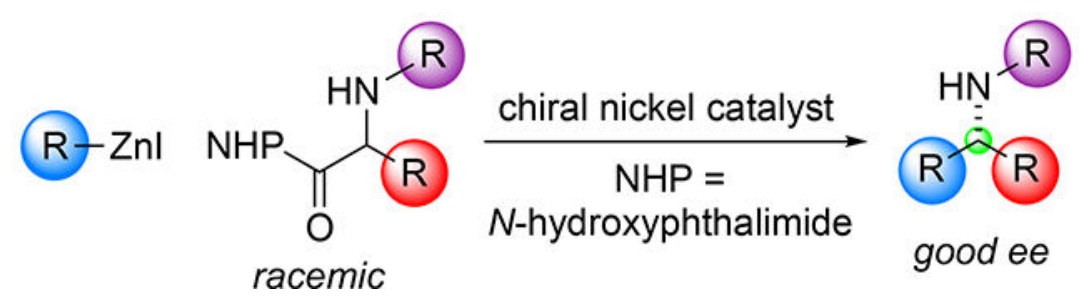

\section{INTRODUCTION}

Because a chiral dialkyl carbinamine subunit is found in a wide array of bioactive molecules (e.g., Figure 1A), the development of efficient methods for its synthesis, particularly catalytic and enantioselective processes, is an important objective in synthetic organic chemistry. ${ }^{1}$ A variety of approaches have been described to date, each of which has limitations,${ }^{2}$ including the addition of alkyl nucleophiles to imines of aliphatic aldehydes (limited scope with respect to the nucleophile), ${ }^{3}$ the reduction/hydrogenation of imines of unsymmetrical dialkylketones (modest enantioselectivity when the alkyl groups are similar) and enamines, ${ }^{4-6}$ and the hydroamination of olefins (modest regioselectivity for many internal olefins). ${ }^{7-9}$ After our study was completed, several groups independently demonstrated that nickel-catalyzed asymmetric reductive couplings of olefins and alkyl halides ${ }^{10}$ can provide access to protected dialkyl carbinamines. ${ }^{11-14}$

With regard to retrosynthetic analysis, the nucleophilic substitution of an alkyl electrophile represents a straightforward approach to the synthesis of dialkyl carbinamines (top of Figure 1B). Although substitution by a nitrogen or by a carbon nucleophile could in principle afford the target molecules, in order to achieve high enantioselectivity, the use of a nitrogen nucleophile would require the effective differentiation between two alkyl groups, whereas the use of a carbon nucleophile would require the effective differentiation between an alkyl group and a nitrogen substituent. We viewed the latter approach to be more likely to provide a general solution to the asymmetric synthesis of dialkyl carbinamines, e.g., for those bearing similar alkyl groups (e.g., $\mathrm{CH}_{2} \mathrm{R}$ versus $\mathrm{CH}_{2} \mathrm{R}^{1}$ ).

Recently, transition metals have been shown to catalyze an array of enantioconvergent couplings of racemic alkyl electrophiles with alkyl nucleophiles. ${ }^{15-18}$ However, there have been no reports of such metal-catalyzed substitution reactions in the case of electrophiles that bear a nitrogen substituent geminal to the leaving group, as required for the strategy for the asymmetric synthesis of dialkyl carbinamines illustrated at the top of Figure 1B. Herein, we describe two complementary approaches to such enantioconvergent substitutions, specifically, nickel-catalyzed couplings of alkylzinc reagents with a-phthalimido alkyl 
chlorides (Method 1) and with $N$-hydroxyphthalimide (NHP) esters of a-amino acids (Method 2).

\section{RESULTS AND DISCUSSION}

\section{Couplings of a-phthalimido alkyl chlorides: Scope.}

The phthalimide functional group is a well-established protected form of a primary amine. ${ }^{19}$ We have determined that a chiral nickel/pybox catalyst can achieve the coupling of an alkylzinc reagent (1.1 equivalents) with a racemic a-phthalimido alkyl chloride to afford a protected dialkyl carbinamine in good yield and enantioselectivity (Figure 2A, entry 1: 90\% yield, $92 \%$ ee). Essentially no alkyl-alkyl bond formation is observed in the absence of $\mathrm{NiBr}_{2}$-glyme or of the pybox ligand (entries 2 and 3), whereas a slightly diminished yield (but good ee) is obtained when half of the standard catalyst loading is used (entry 4). The presence of water or of air impedes carbon-carbon bond formation, while the enantioselectivity is not affected (entries 5 and $6^{20}$ )(for the impact of other reaction parameters, see Section VI of the Supporting Information).

As illustrated in Figure 2B.1, the scope of this method for the catalytic enantioconvergent synthesis of protected dialkyl carbinamines is fairly broad with respect to the electrophile. For example, good yields and ee's are observed when alkyl substituent $\mathrm{R}$ varies in size from methyl to isobutyl (products 1-4), although a poor yield is observed if it is a bulky isopropyl group. A variety of functional groups are compatible with the method, including an aryl iodide, ester, carbonate, unactivated primary alkyl halide (fluoride, chloride, and bromide), indazole, and activated heteroaryl chloride (products 5-14). In the case of an electrophile that bears a remote stereocenter, the stereochemistry of the catalyst, rather than that of the substrate, controls the stereochemistry of the product (products $\mathbf{1 5}$ and 16). On a gram-scale (1.40 $\mathrm{g}$ of product), the coupling to generate product 2 proceeds in similar yield and ee (93\% yield, $92 \%$ ee) as for a reaction conducted on a $0.6-\mathrm{mmol}$ scale (94\% yield, $92 \%$ ee).

The scope of this enantioconvergent alkyl-alkyl coupling is also broad with respect to the nucleophile, leading to an array of protected dialkyl carbinamines with good yield and ee. For example, the $\mathrm{R}$ substituent can range in size from $n$-hexyl to isobutyl (Figure 2B.2, products 17-19; however, the use of a secondary alkylzinc reagent results in a low yield of the coupling product), and a variety of functional groups can be present (entries 20-35; for additional studies of the functional-group compatibility of the method, see the Supporting Information).

\section{Couplings of a-phthalimido alkyl chlorides: Mechanistic observations.}

We have previously reported that two distinct nickel-catalyzed enantioconvergent couplings (Negishi reactions of propargylic halides and Kumada reactions of a-haloketones) appear to proceed through a common pathway (Figure 3A), wherein the predominant resting state of the catalyst is an organonickel(II) complex (A). ${ }^{21,22}$ For the couplings of a-phthalimido alkyl chlorides with alkylzinc reagents described herein, our mechanistic observations are again consistent with this pathway. 
For example, quantitative EPR analysis indicates that odd-electron nickel intermediates (e.g., $\mathrm{Ni}^{\mathrm{I}}$ or $\left.\mathrm{Ni}^{\mathrm{III}}\right)$ do not accumulate to a significant extent during the reaction $(<2 \%$ of the total nickel present). Furthermore, ESI-MS analysis of a coupling (Figure 2A) at partial conversion reveals masses consistent with $\mathbf{A}^{\mathbf{1}}$ and $\mathbf{A}^{\mathbf{2}}$ (Figure 3B). Finally, when the same coupling is conducted in the presence of TEMPO, a TEMPO adduct of the electrophile can be isolated (Figure 3C), consistent with the generation of an organic radical from the alkyl chloride.

\section{Couplings of NHP esters of $a$-amino acids: Scope.}

Redox-active esters (e.g., NHP esters) serve as useful partners in a variety of metal-catalyzed carbon-carbon bond-forming reactions. ${ }^{23-27}$ The use of NHP esters derived from readily available a-amino acids ${ }^{28-32}$ could provide a complementary strategy to the use of a-amino halides, many of which are relatively unstable, to generate an organic radical (Figure 3A) en route to enantioenriched dialkyl carbinamines.

After an extensive survey of reaction parameters, we determined that the desired decarboxylative coupling of a racemic NHP ester with an alkylzinc reagent can be achieved in the presence of a chiral nickel/diamine catalyst, providing the $N$-protected dialkyl carbinamine in good yield and ee (Figure 4A, entry 1; 79\% yield, 91\% ee). It is worth noting that only 1.2 equivalents of the nucleophile are used, despite the presence of a potentially labile N-H proton; in contrast, most previous metal-catalyzed couplings of NHP esters have employed at least 2 equivalents of the organometallic nucleophile, even in the absence of an acidic proton. ${ }^{23,24}$

Essentially no carbon-carbon bond formation is observed in the absence of $\mathrm{NiBr}_{2}$.glyme (Figure 4A, entry 2), and the coupling proceeds in significantly lower yield and/or ee when chiral diamine $\mathbf{L 2}, \mathrm{LiCl}^{33,34} \mathrm{TMSCl}^{35,36}$ or DMAP ${ }^{37}$ is omitted (entries 3-6). The use of half of the standard catalyst loading results in a small loss in efficiency (entry 7;65\% yield, $88 \%$ ee). From a practical point of view, it is noteworthy that this enantioconvergent coupling is not highly water- or air-sensitive: the addition of 0.05 equivalents of water or of $1 \mathrm{~mL}$ of air to the reaction vessel has only a minor deleterious effect (entries 8 and 9)(for the impact of other reaction parameters, see Section VI of the Supporting Information).

A variety of NHP esters serve as suitable coupling partners in these nickel-catalyzed enantioconvergent couplings to generate protected dialkyl carbinamines (Figure 4B.1 and 4B.2). The alkyl group $\mathrm{R}$ can vary in steric demand from Me to $i$-Pr (products 36-40), and it can bear a range of functional groups, including a thioether, an indole, and a thiophene (products 41-48). The method can be applied to glutamic acid and proline derivatives, thereby affording enantioenriched protected $\gamma$-amino acids ${ }^{38,39}$ and 2-alkylpyrrolidines ${ }^{40,41}$ in good ee from readily available starting materials (products $\mathbf{4 7}$ and $\mathbf{4 8}$ ). Not only Bocprotected, but also Fmoc- and Cbz-protected, amines are useful reaction partners (products 49 and 50). The coupling products are generally crystalline, allowing ready enhancement of stereochemical purity (e.g., products $\mathbf{5 1}$ and 69).

The scope of this method is also broad with respect to the nucleophile (Figure 4B.3). Unbranched and branched primary (but not secondary) alkylzinc reagents serve as suitable 
nucleophiles (products 51-54), as do a variety of functionalized alkylzincs (products 55$\mathbf{7 2} ;{ }^{42}$ see the Supporting Information for additional functional-group compatibility studies).

This approach to the catalytic asymmetric synthesis of protected dialkyl carbinamines can be achieved in a one-pot process without isolation of the NHP ester, ${ }^{43}$ thereby providing the desired products in one step from commercially available protected $a$-amino acids (Figure 5A). The yields for the one-pot procedure are similar to or modestly lower than for the corresponding couplings of purified NHP esters, and the enantioselectivities are essentially identical. The success of this process is a testament to the robustness of the methodimpurities and side products from the DIC coupling, including $N, N$-diisopropylurea, neither poison the catalyst nor consume the alkylzinc reagent via protonation, enabling the reaction to proceed with only 1.2 equivalents of the nucleophile. ${ }^{44}$

\section{Couplings of NHP esters of a-amino acids: Applications.}

We have applied our catalytic asymmetric synthesis of protected dialkyl carbinamines to a variety of target molecules, starting from commercially available a-amino acid derivatives (Figure 5B). For example, urea 74, an analog of an inhibitor of protein kinases 1 and $2,{ }^{45}$ can be synthesized in two steps and $40 \%$ overall yield from $N$-Boc-alanine, via a one-pot coupling followed by conversion of the carbamate to the urea. Furthermore, Fmoc-protected aminoalcohol 75, an intermediate in the synthesis of a constrained peptidomimetic (prior route: eight steps), ${ }^{46}$ can be produced in two steps from $N$-Fmoc-phenylalanine using our method; although the nickel-catalyzed coupling itself proceeds with moderate enantioselectivity ( $81 \%$ ee), Fmoc-protected aminoalcohol 75 can readily be recrystallized to $>99 \%$ ee. Pyrrolidine 76, which has previously been generated in four steps from $\mathrm{N}$-Cbzproline en route to a hydrazone-based chiral auxiliary, ${ }^{47}$ can be synthesized in one pot and $72 \%$ yield from $N$-Boc-proline via our approach. Finally, pyrrolidine 78, which has been employed as an intermediate in a study of serotonin inhibitors, can be formed in $50 \%$ overall yield in three, rather than eight, steps, via a nickel-catalyzed coupling. ${ }^{48}$

\section{Couplings of NHP esters of a-amino acids: Mechanistic observations:}

Our working hypothesis is that these nickel-catalyzed enantioconvergent couplings of NHP esters may be following a pathway analogous to that outlined in Figure 3A for couplings of alkyl halides, wherein the same radical $\mathrm{R} \bullet$ may be generated by the decarboxylative reduction of the NHP ester by LXNiI ${ }^{23,49}$ As in the case of couplings of a-phthalimido alkyl chlorides (see above), the EPR spectrum of the nickel-catalyzed reaction of the NHP ester illustrated in Figure 4A indicates that odd-electron nickel intermediates do not accumulate to a significant extent during the coupling ( $<2 \%$ of the total nickel present). Furthermore, $\mathrm{C}-\mathrm{C}$ bond formation is inhibited by the presence of TEMPO. ${ }^{50}$

We have examined whether the chiral nickel catalyst achieves any kinetic resolution in the enantioconvergent coupling of a racemic NHP ester. Although this issue has been explored in the case of alkyl halides, ${ }^{51,52}$ we are not aware of corresponding investigations in the case of NHP esters. When the coupling of a racemic NHP ester is stopped at partial conversion, the unreacted NHP ester is still racemic $(<1 \%$ ee; Figure $5 \mathrm{C}$, experiment 1$)$. Taken together with our observation that enantioenriched NHP ester does not racemize under the reaction 
conditions (experiment 2), these data indicate that the chiral nickel catalyst is reacting at essentially identical rates with each enantiomer of the NHP ester (no kinetic resolution).

\section{CONCLUSIONS}

We have developed two versatile methods for the catalytic asymmetric synthesis of dialkyl carbinamines, an important family of molecules in chemistry and biology, through the use of chiral catalysts based on nickel, an earth-abundant metal. With an alkylzinc reagent (1.1-1.2 equivalents) as the nucleophile, enantioconvergent couplings can be achieved under mild conditions with either an a-phthalimido alkyl chloride or an NHP ester of a protected aamino acid; both methods display broad scope and good functional-group tolerance. The NHP esters can be generated in situ from commercially available $a$-amino acid derivatives and coupled directly, resulting in a straightforward one-pot catalytic enantioselective synthesis of a variety of interesting target molecules.

\section{Supplementary Material}

Refer to Web version on PubMed Central for supplementary material.

\section{ACKNOWLEDGMENTS}

Support has been provided by the National Institutes of Health (National Institute of General Medical Sciences; grant R01-GM062871), the National Science Foundation Graduate Research Fellowship Program (grant DGE-1745301 to D. J. F.), and the Dow Next-Generation Educator Fund (grant to Caltech). We thank Dr. Haohua Huo for important early contributions to this project, and we thank Nicholas J. Fastuca, Lawrence M. Henling and Dr. Michael K. Takase (Caltech X-Ray Crystallography Facility), Dr. Paul H. Oyala (Caltech EPR Facility), Dr. Felix Schneck, Xiaoyu Tong, Dr. David G. VanderVelde (Caltech NMR Facility), Dr. Scott C. Virgil (Caltech Center for Catalysis and Chemical Synthesis), and Wanji Zhang for assistance and helpful discussions.

\section{REFERENCES}

(1). For an overview, see: Nugent TC Chiral Amine Synthesis: Methods, Developments and Applications; Wiley-VCH: Wyeinheim, 2010.

(2). For leading references, see: Yin Q; Shi Y; Wang J; Zhang X Direct catalytic asymmetric synthesis of a-chiral primary amines. Chem. Soc. Rev. 2020, 49, 6141-6153. [PubMed: 32666962]

(3). For a review, see: Lindsay VNG; Charette AB Nucleophilic Addition of Nonstabilized Carbanions to Imines and Imine Derivatives. Comprehensive Organic Synthesis, 2nd Edition; Elsevier: Amsterdam, Netherlands, 2014; Vol. 1, pp. 365-394.

(4). For overviews, see: Ponra S; Boudet B; Phansavath P; Ratovelomanana-Vidal V Recent Developments in Transition-Metal-Catalyzed Asymmetric Hydrogenation of Enamides. Synthesis 2021, 53, 193-214.

(5). Wang C; Xiao J Asymmetric Reductive Amination. Top. Curr. Chem. 2014, 343, 261-282. [PubMed: 24158548]

(6). Xie J-H; Zhu S-F; Zhou Q-L Transition Metal-Catalyzed Enantioselective Hydrogenation of Enamines and Imines. Chem. Rev. 2011, 111, 1713-1760. [PubMed: 21166392]

(7). Michon C; Abadie M-A; Medina F; Agbossou-Niedercorn F Recent metal-catalysed asymmetric hydroaminations of alkenes. J. Organomet. Chem. 2017, 847, 13-27.

(8). Reference 1, pp. 341-375.

(9). Xi Y; Ma S; Hartwig JF Catalytic asymmetric addition of an amine N-H bond across internal alkenes. Nature 2020, 588, 254-260. [PubMed: 33142305]

(10). Wang Z; Yin H; Fu GC Catalytic Enantioconvergent Coupling of Secondary and Tertiary Electrophiles with Olefins. Nature 2018, 563, 379-383. [PubMed: 30337711] 
(11). Qian D; Bera S; Hu X Chiral Alkyl Amine Synthesis via Catalytic Enantioselective Hydroalkylation of Enamides. ChemRxiv, 2020, https://chemrxiv.org/articles/preprint/Chiral_Alkyl_Amine_Synthesis_via_Catalytic_Enantioselective_Hydroalkylation_of_Enamides/ 13096121/1 (most ee's <90\%).

(12). Wang J-W; Li Y; Nie W; Chang Z; Yu Z-A; Zhao Y-F; Lu X; Fu Y Catalytic asymmetric reductive alkylation of enamines to chiral aliphatic amines. ChemRxiv, 2020, https:// chemrxiv.org/articles/preprint/

Catalytic_Asymmetric_Reductive_Alkylation_of_Enamines_to_Chiral_Aliphatic_Amines/ 13102307.

(13). Wang S; Zhang T-Y; Zhang J-X; Meng H; Chen B-H; Shu W Enantioselective Access to Dialkyl Amines and Alcohols via Ni-Catalyzed Reductive Hydroalkylations. ChemRxiv, 2020, https:// chemrxiv.org/articles/preprint/Enantioselective_Access_to_Dialkyl_Amines_and_Alcohols_via_Ni-Catalyzed_Reductive_Hydroalkylations/13284416 (limited to methyl alkyl carbinamines).

(14). See also: Schmidt J; Choi J; Liu AT; Slusarczyk M; Fu GC A General, Modular Method for the Catalytic Asymmetric Synthesis of Alkylboronate Esters. Science 2016, 354, 1265-1269. [PubMed: 27940868]

(15). Choi J; Fu GC Transition metal-catalyzed alkyl-alkyl bond formation: Another dimension in cross-coupling chemistry. Science 2017, 356, eaaf7230. [PubMed: 28408546]

(16). Fu GC Transition-Metal Catalysis of Nucleophilic Substitution Reactions: A Radical Alternative to SN1 and SN2 Processes. ACS Cent. Sci. 2017, 3, 692-700. [PubMed: 28776010]

(17). Kaga A; Chiba S Engaging Radicals in Transition Metal-Catalyzed Cross-Coupling with Alkyl Electrophiles: Recent Advances. ACS Catal. 2017, 7, 4697-4706.

(18). Iwasaki T; Kambe N Ni-Catalyzed C-C Couplings Using Alkyl Electrophiles. Top. Curr. Chem. 2016, 374, 66.

(19). For example, see: Ahmad NM Gabriel synthesis. Name Reactions for Functional Group Transformations; Li JJ, Corey EJ, Eds.; John Wiley \& Sons: Hoboken, NJ, 2007; pp. 438-450.

(20). When the reaction time was increased for entry 6 , the yield increased to $77 \%$ (91\% ee).

(21). Schley ND; Fu GC Nickel-Catalyzed Negishi Arylations of Propargylic Bromides: A Mechanistic Investigation. J. Am. Chem. Soc. 2014, 136, 16588-16593. [PubMed: 25402209]

(22). Yin H; Fu GC Mechanistic Investigation of Enantioconvergent Kumada Reactions of Racemic aBromoketones Catalyzed by a Nickel/Bis(oxazoline) Complex. J. Am. Chem. Soc. 2019, 141, 15433-15440. [PubMed: 31502449]

(23). For an example of the use of redox-active esters in nickel-catalyzed cross-couplings, see: Cornella J; Edwards JT; Qin T; Kawamura S; Wang J; Pan C-M; Gianatassio R; Schmidt M; Eastgate MD; Baran PS Practical Ni-Catalyzed Aryl-Alkyl Cross-Coupling of Secondary RedoxActive Esters. J. Am. Chem. Soc. 2016, 138, 2174-2177. [PubMed: 26835704]

(24). For an overview of the use of redox-active esters in coupling reactions, see: Murarka S N(Acyloxy)phthalimides as Redox-Active Esters in Cross-Coupling Reactions. Adv. Synth. Catal. 2018, 360, 1735-1753.

(25). For reports of metal-catalyzed enantioconvergent coupling reactions of redox-active esters, see: reductive cross-coupling: Suzuki N; Hofstra JL; Poremba KE; Reisman SE Nickel-Catalyzed Enantioselective Cross-Coupling of $\mathrm{N}$-Hydroxyphthalimide Esters with Vinyl Bromides. Org. Lett. 2017, 19, 2150-2153. [PubMed: 28375631]

(26). Wang D; Zhu N; Chen P; Lin Z; Liu G Enantioselective Decarboxylative Cyanation Employing Cooperative Photoredox Catalysis and Copper Catalysis. J. Am. Chem. Soc. 2017, 139, 1563215635. [PubMed: 29039930]

(27). Xia H-D; Li Z-L; Gu Q-S; Dong X-Y; Fang J-H; Du X-Y; Wang L-L; Liu X-Y Photoinduced Copper-Catalyzed Asymmetric Decarboxylative Alkynylation with Terminal Alkynes. Angew. Chem. Int. Ed. 2020, 59, 16926-16932.

(28). For examples of metal-catalyzed coupling reactions of redox-active esters of a-amino acids with organometallic nucleophiles (none are enantioselective), see: Qin T; Cornella J; Li C; Malins LR; Edwards JT; Kawamura S; Maxwell BD; Eastgate MD; Baran PS A general alkyl-alkyl cross- 
coupling enabled by redox-active esters and alkylzinc reagents. Science 2016, 352, 801-805 (1 example). [PubMed: 27103669]

(29). Toriyama F; Cornella J; Wimmer L; Chen T-G; Dixon DD; Creech G; Baran PS Redox-Active Esters in Fe-Catalyzed C-C Coupling. J. Am. Chem. Soc. 2016, 138, 11132-11135 (3 examples). [PubMed: 27548696]

(30). Liu X-G; Zhou C-J; Lin E; Han X-L; Zhang S-S; Li Q; Wang H Decarboxylative Negishi Coupling of Redox-Active Aliphatic Esters by Cobalt Catalysis. Angew. Chem. Int. Ed. 2018, 57, 13096-13100 (4 examples).

(31). Wang Z-Z; Wang G-Z; Zhao B; Shang R; Fu Y Cobalt-Catalyzed Decarboxylative Methylation and Ethylation of Aliphatic N-(Acyloxy)phthalimides with Organoaluminum Reagents. Synlett 2020, 31, 1221-1225 (1 example).

(32). For a nickel-catalyzed enantioselective decarboxylative coupling of an a-amino acid with an aryl halide, see: Zuo Z; Cong H; Li W; Choi J; Fu GC; MacMillan DWC Enantioselective Decarboxylative Arylation of a-Amino Acids via the Merger of Photoredox and Nickel Catalysis. J. Am. Chem. Soc. 2016, 138, 1832-1835. [PubMed: 26849354]

(33). For an early report of the beneficial effect of a halide salt on an enantioconvergent coupling reaction, see: Son S; Fu GC Nickel-Catalyzed Asymmetric Negishi Cross-Couplings of Secondary Allylic Chlorides with Alkylzincs. J. Am. Chem. Soc. 2008, 130, 2756-2757. [PubMed: 18257579]

(34). For a recent discussion of salt effects in Negishi reactions, see: Eckert P; Sharif S; Organ MG Salt to Taste: The Critical Roles Played by Inorganic Salts in Organozinc Formation and in the Negishi Reaction. Angew. Chem. Int. Ed. doi.org/10.1002/anie.202010917.

(35). The beneficial effect of TMSCl may arise from the removal of $\mathrm{N}$-zincated phthalimide that is formed during the coupling process. Under our standard conditions, we have identified resonances in the $1 \mathrm{H}$ and $29 \mathrm{Si} \mathrm{NMR}$ spectra that match independently synthesized Ntrimethylsilylphthalimide.

(36). TMSBr has previously been shown to be beneficial in an enantioselective cross-electrophile coupling: Suzuki N; Hofstra JL; Poremba KE; Reisman SE Nickel-Catalyzed Enantioselective Cross-Coupling of N-Hydroxyphthalimide Esters with Vinyl Bromides. Org. Lett. 2017, 19, 2150-2153. [PubMed: 28375631] The authors note that TMSCl was less effective and that the role of TMSBr is "unclear".

(37). Our discovery of the beneficial effect of DMAP arose from our investigation of a one-pot coupling procedure through the use of NHP esters that are generated in situ (in the presence of DIC and DMAP; Figure 5A). If a hindered pyridine (2,6-dimethylpyridine) is employed instead of DMAP, no enhancement in enantioselectivity is observed. For a previous example of improved enantioselectivity in the presence of pyridine itself, see: Zhao D; Mao L; Yang D; Wang R ZincMediated Asymmetric Additions of Dialkylphosphine Oxides to a, $\beta$-Unsaturated Ketones and $N$ Sulfinylimines. J. Org. Chem. 2010, 75, 6756-6763. [PubMed: 20845966]

(38). For an overview and leading references, see: Ordóñez M; Cativiela C; Romero-Estudillo I An update on the stereoselective synthesis of $\gamma$-amino acids. Tetrahedron: Asymmetry 2016, 27 , 999-1055.

(39). Al-Majed A Vigabatrin. Profiles of Drug Substances, Excipients, and Related Methodology; Brittain HG, Ed.; Elsevier: Amsterdam, Netherlands, 2010; Vol. 35, pp. 309-345.

(40). For overviews and leading references, see: Vega-Peñaloza A; Paria S; Bonchio M; Dell'Amico L; Companyó X Profiling the Privileges of Pyrrolidine-Based Catalysts in Asymmetric Synthesis: From Polar to Light-Driven Radical Chemistry. ACS Catalysis 2019, 9, 6058-6072.

(41). Bhat C; Tilve SG Recent advances in the synthesis of naturally occurring pyrrolidines, pyrrolizidines and indolizidine alkaloids using proline as a unique chiral synthon. RSC Adv. $2014,4,5405-5452$.

(42). The lower stereoselectivity for products 69 and 70 may be due to an interaction of the carbonyl oxygen of the ester with the chiral nickel catalyst (six-membered chelate) in the stereochemistrydetermining step of the reaction.

(43). For a prior example of one-pot couplings of NHP esters that are generated in situ, see: Edwards JT; Merchant RR; McClymont KS; Knouse KW; Qin T; Malins LR; Vokits B; Shaw SA; Bao DH; Wei F-L; Zhou T; Eastgate MD; Baran PS Decarboxylative alkenylation. Nature 2017, 545, 
213-219. [PubMed: 28424520] In this study, the standard coupling conditions employ 2.0 equivalents of the nucleophile, whereas the one-pot procedure employs 3.0 equivalents of the nucleophile, "due to the acidic protons of the DIC urea byproduct".

(44). Prior couplings of NHP esters that are generated in situ have nearly always employed a significant excess of the nucleophile (at least 2 equivalents). For example, see reference 43.

(45). Lackey K; Schnellmann R; Goodwin AJ Treatment of Septicemia and ARDS with ERK Inhibitors. PCT Int. Appl. WO2017180817 (A1), 2017.

(46). Nasopoulou M; Georgiadis D; Matziari M; Dive V; Yiotakis A A Versatile Annulation Protocol toward Novel Constrained Phosphinic Peptidomimetics. J. Org. Chem. 2007, 72, 7222-7228. [PubMed: 17715974]

(47). Denmark SE; Edwards JP; Weber T; Piotrowski DW Organocerium additions to proline-derived hydrazones: synthesis of enantiomerically enriched amines. Tetrahedron: Asymmetry 2010, 21, 1278-1302.

(48). Tanaka N; Goto R; Ito R; Hayakawa M; Sugidachi A; Ogawa T; Asai F; Fujimoto K [2-( $\omega$ Phenylalkyl)phenoxy]alkylamines II: Synthesis and Selective Serotonin-2 Receptor Binding. Chem. Pharm. Bull. 2000, 48, 245-255.

(49). Oelke AJ; Sun J; Fu GC Nickel-Catalyzed Enantioselective Cross-Couplings of Racemic Secondary Electrophiles That Bear an Oxygen Leaving Group. J. Am. Chem. Soc. 2012, 134, 2966-2969. [PubMed: 22296603]

(50). Although we have not been able to isolate the TEMPO adduct of the postulated organic radical, perhaps due to its lability, we have confirmed its presence through high-resolution mass spectrometry.

(51). For an example wherein kinetic resolution is observed, see: Lundin PM; Fu GC Asymmetric Suzuki Cross-Couplings of Activated Secondary Alkyl Electrophiles: Arylations of Racemic aChloroamides. J. Am. Chem. Soc. 2010, 132, 11027-11029. [PubMed: 20698665]

(52). For an example wherein kinetic resolution is not observed, see: Fischer C; Fu GC Asymmetric Nickel-Catalyzed Negishi Cross-Couplings of Secondary a-Bromo Amides with Organozinc Reagents. J. Am. Chem. Soc. 2005, 127, 4594-4595. [PubMed: 15796523] 


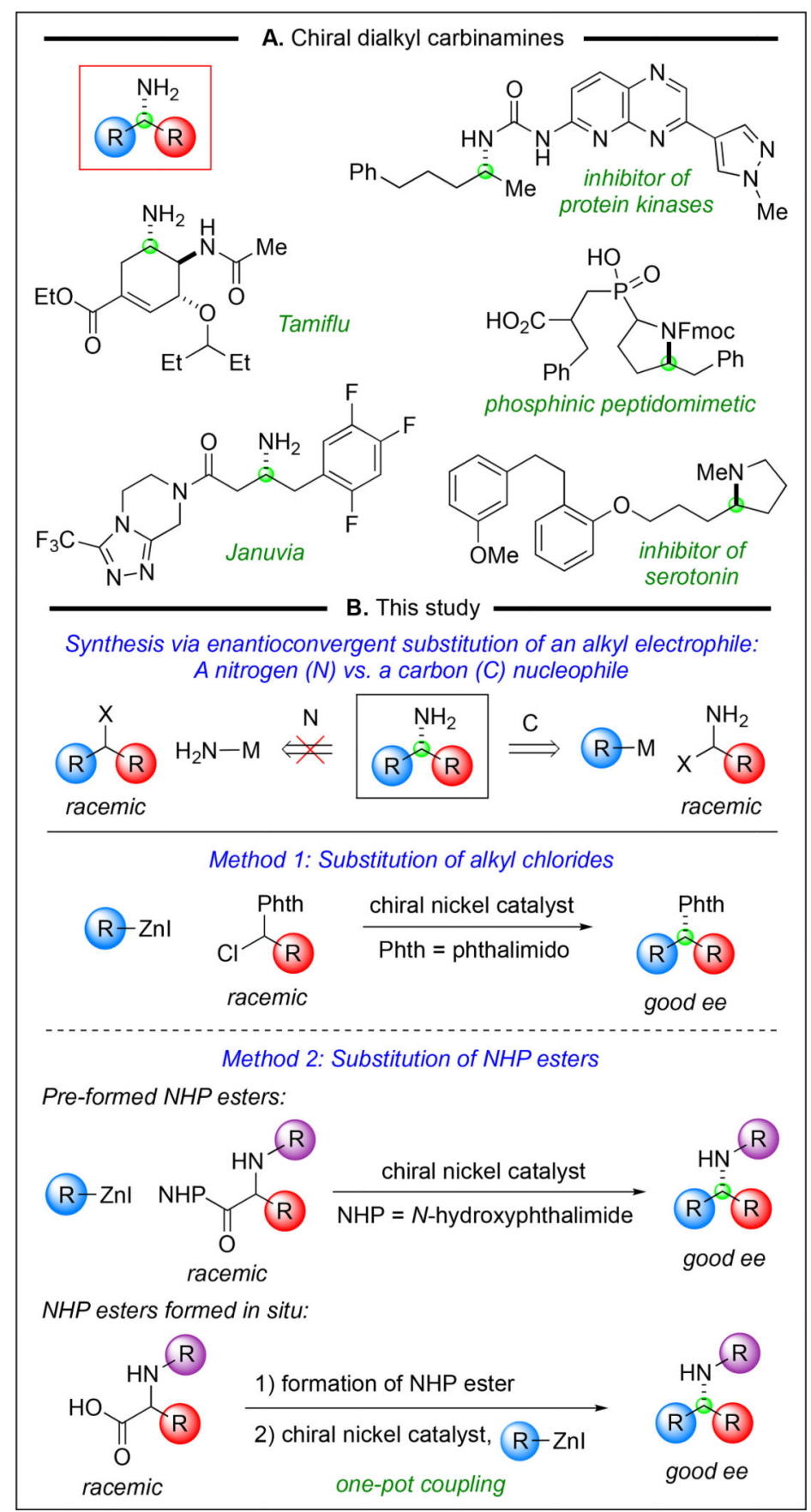

Figure 1.

Dialkyl carbinamines. (A) Examples of compounds that include a chiral dialkyl carbinamine subunit. (B) This study: Nickel-catalyzed enantioconvergent substitution reactions of alkyl electrophiles to generate protected dialkyl carbinamines. 


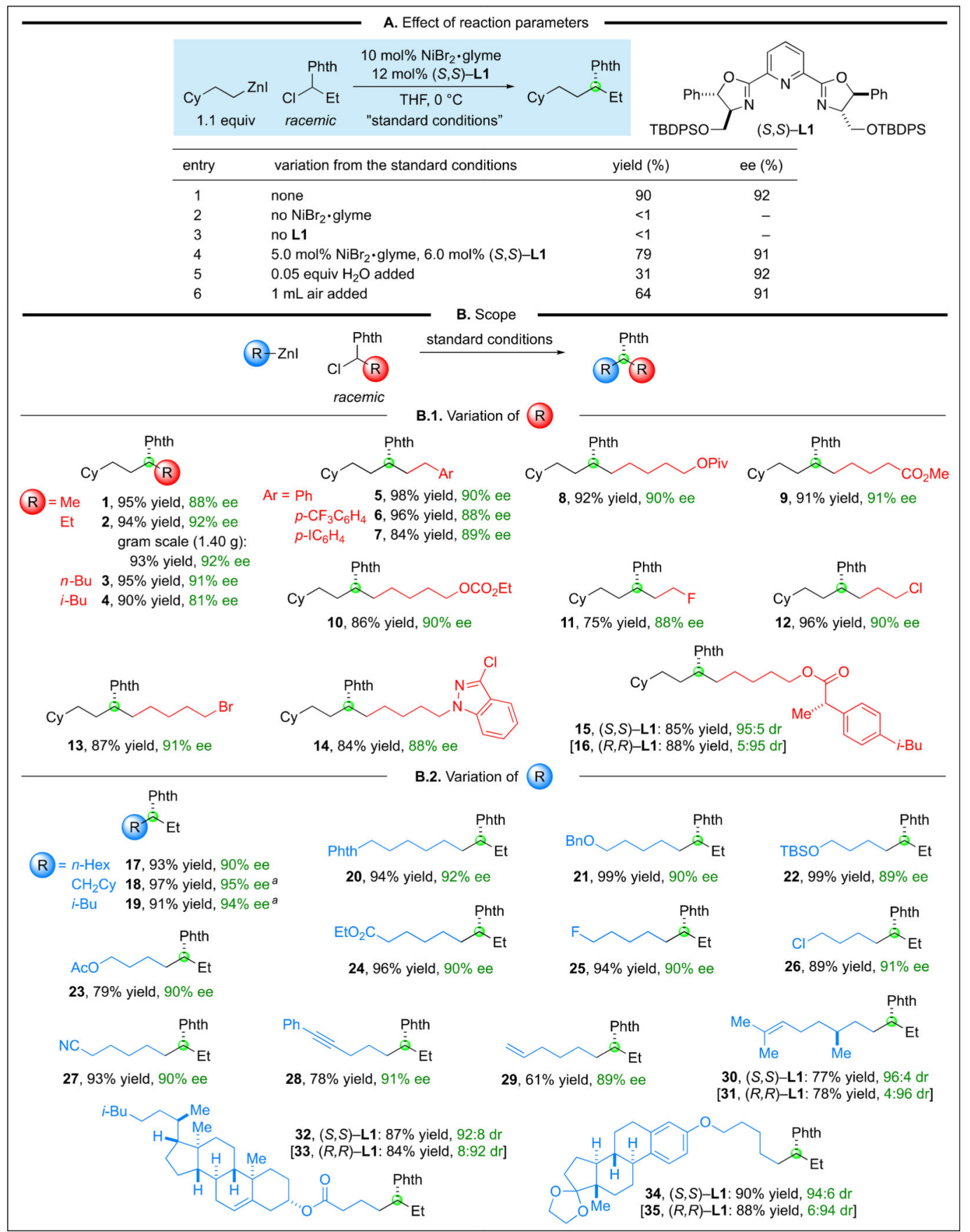

Figure 2.

Enantioconvergent substitution reactions of alkyl chlorides to generate phthalimideprotected dialkyl carbinamines. (A) Effect of reaction parameters. (B) Scope. All data are the average of two experiments run on a $0.6-\mathrm{mmol}$ scale (unless otherwise noted), and all yields are of purified products. ${ }^{a}$ The reaction was conducted at r.t. 

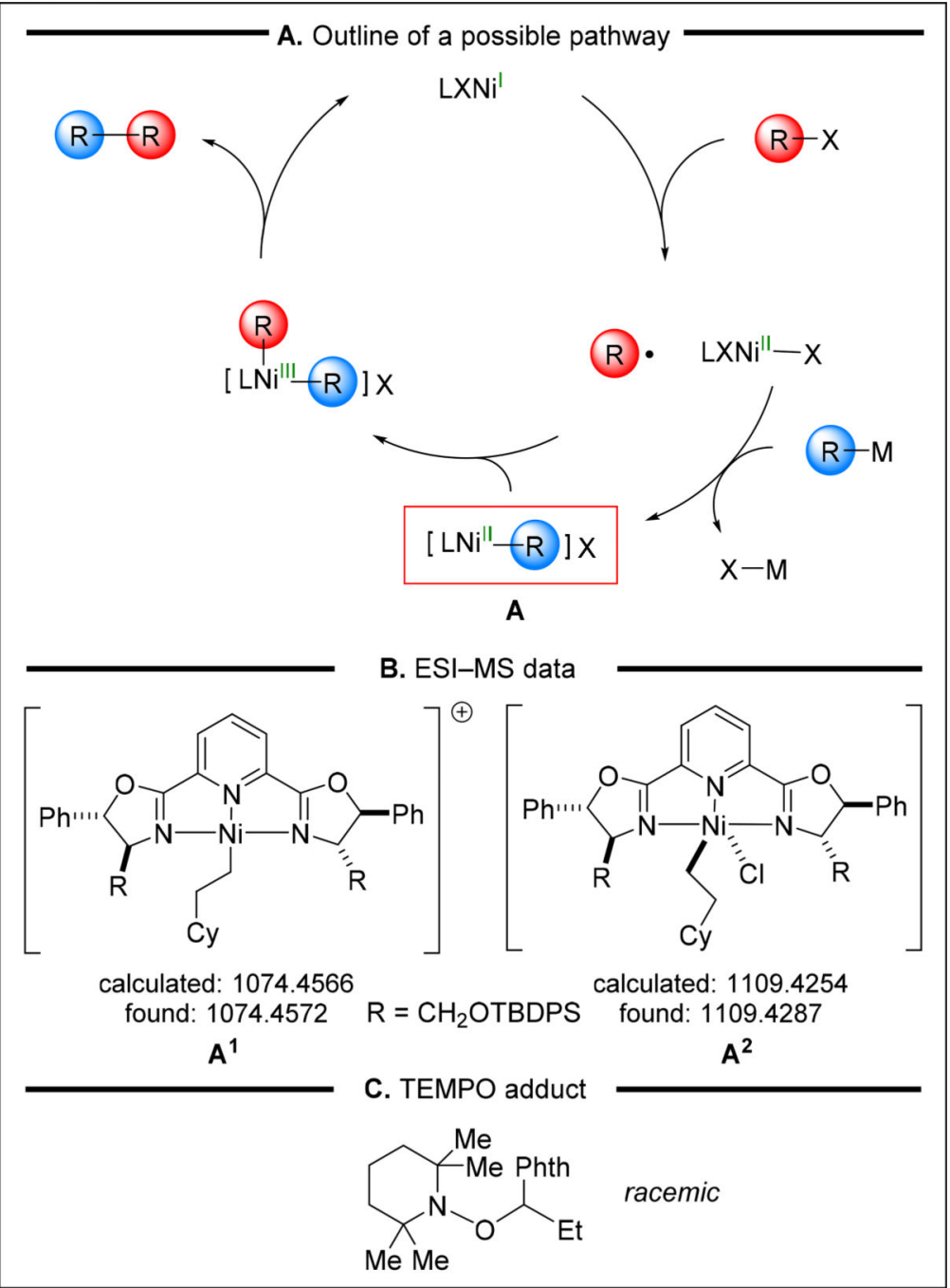

Figure 3.

Nickel-catalyzed enantioconvergent substitution reactions: Mechanism. (A) Outline of a possible pathway. (B) ESI-MS data for the coupling illustrated in Figure 2A. (C) TEMPO adduct of the electrophile (Figure $2 \mathrm{~A}$ ). $\mathrm{X}=$ halide (an inner- or an outer-sphere ligand). 


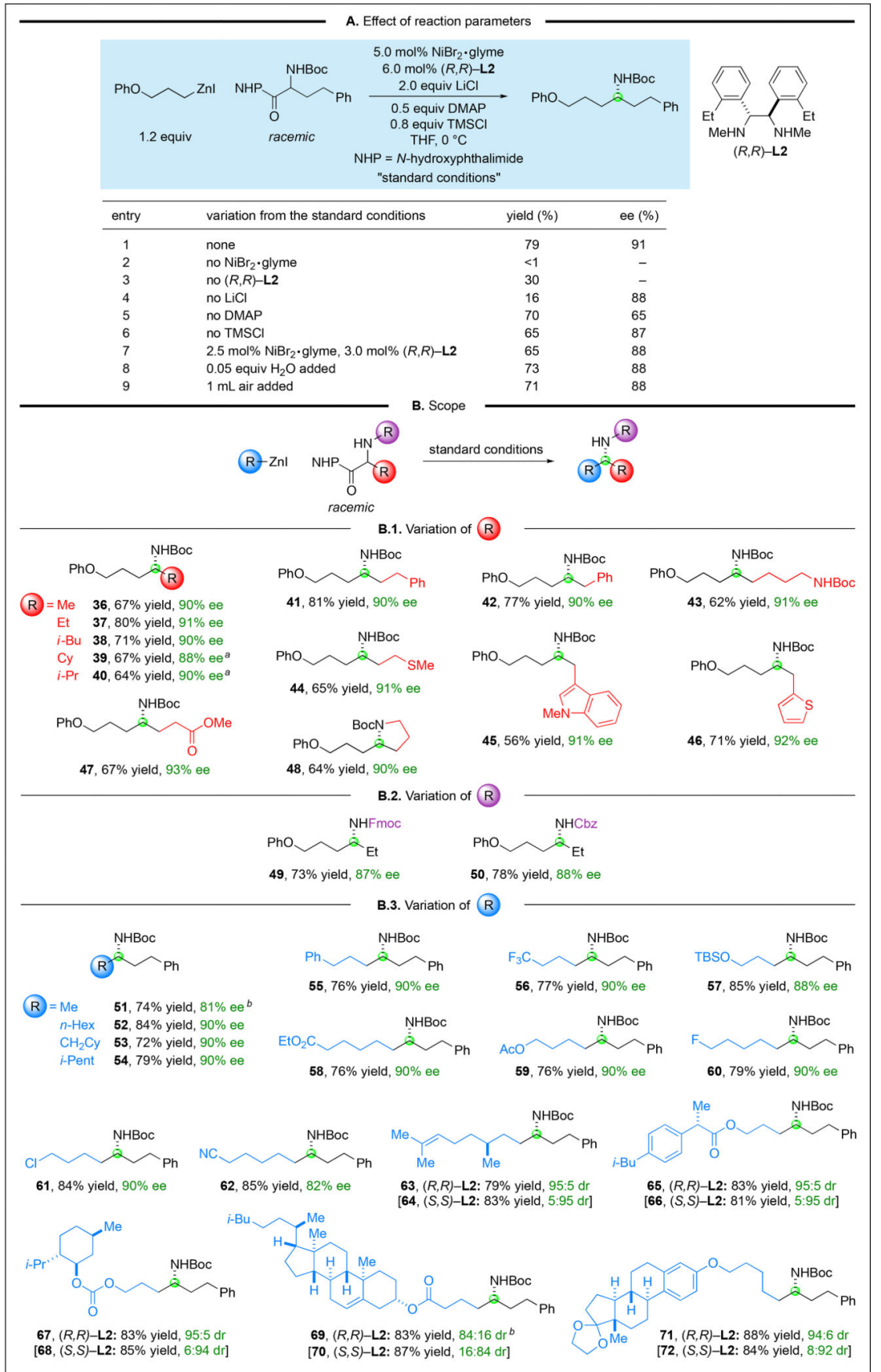

Figure 4.

Enantioconvergent synthesis of protected dialkyl carbinamines from racemic NHP esters. (A) Effect of reaction parameters. (B) Scope. All data are the average of two experiments run on a $0.6-\mathrm{mmol}$ scale, and all yields are of purified products. ${ }^{a} 10 \mathrm{~mol} \% \mathrm{NiBr}_{2} \cdot \mathrm{glyme}, 12$ $\mathrm{mol} \% \mathbf{L 2}$, and 5.0 equiv $\mathrm{LiCl}$ were used (no DMAP or TMSCl). ${ }^{b}$ The product was recrystallized to $>99 \%$ ee or $>99.5: 0.5$ d.r. 


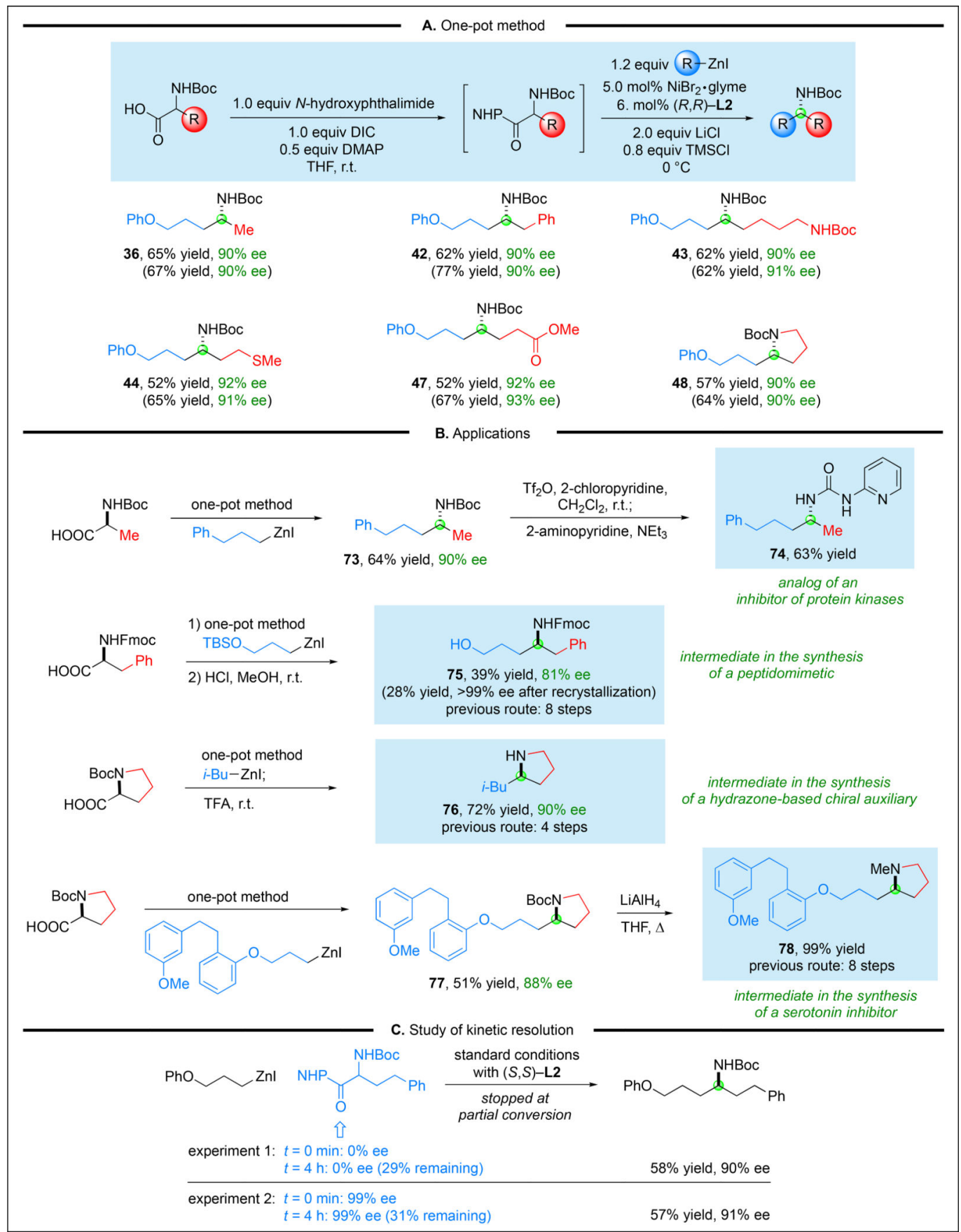

Figure 5.

Asymmetric synthesis of protected dialkyl carbinamines via substitution reactions of NHP esters. (A) One-pot procedure. The values in parentheses are the data for the corresponding couplings of purified NHP esters (see Figure 4B). (B) Applications. (C) Study of kinetic resolution. 